\title{
Study on Reconstruction of Spectral Reflectance with HIS
}

\author{
Xiao-min Zhang ${ }^{*}{ }^{1}$, Song-hua $\mathrm{He}^{2^{*}}$, Qiao Chen $^{3}$ \\ 1.School of Media and Design,Hangzhou Dianzi University \\ 2. Department of Communication Engineering, Shen Zhen Polytechnic \\ 3.School of Media and Design, Hangzhou Dianzi University
}

Keywords: Hyperspectral Imaging System (HIS), Spectral Reflectance, Relative Colorimetric Assay, Dark Field Correction

\begin{abstract}
This paper take reconstruction of spectral reflectance use Hyper-spectral Imaging System (HIS) as the main research object. A new method based on the relative colorimetric assay and dark field correction for spectral reflectance reconstructing be proposed to meet the need of high fidelity color reproduction(Hi-Fi) after test the property and the shooting parameters comprehensively. The principle and application of the method above are introduced thoroughly in this paper and be used to reconstruct the spectral reflectance of hyper-spectral imaging system. The performance of method above and flat-field method demonstrated that the method this paper proposed is better whether colorimetric or spectrum similarity.
\end{abstract}

\section{Introduction}

Since spectral imaging technology can be used to record the image scene in spectral way that reflects the surface color characteristic of objects, this technology has found wide application in remote sensing, agriculture, $\mathrm{Hi}-\mathrm{Fi}$ and so on. Spectral radiation intensity can be sampled in different wavelength interval as multi-spectral images that conclude position and radiation intensity in every sampled wavelength with Spectral Imaging Technology .This technology has been successfully applied in the HIS and it's sample interval commonly is nano-scale .In order to use the images that captured by HIS in field of Hi-Fi, we must find an appropriate reflectance reconstruction method to transform the radiation intensity into the spectral reflectance. There are two kinds of reflectance reconstruction methods have been proposed with transfer function and image structure respectively and the method called flat-field belongs to the latter method is more typical. Comparative colorimetric assay method and dark field correction method are combined to reconstruct spectral reflectance after obtained the best parameters in the process of image capturing, and then compared the experimental results with flat-field. The comparisons indicated that the method proposed in this paper can obtain higher reconstructive precision both in colorimetric and spectrum comparability, which verifies the method we proposed has more practicability in Hi-Fi.

\section{Spectral Reflectance Reconstruction Method with HSI}

\section{Flat-field}

Flat-field find a flat area in the captured image with high brightness, large area, uniform surface and most importantly is the curve of spectrum in this part must be smooth enough. And then the HIS response of the whole image divided by the HIS response of the flat area we can calculate the spectral reflectance of every pixel in this image. Flat-field can minimise the inhomogeneity of image caused by uneven lighting source, camera lens, image sensor or other environmental factors. This reconstruction method mainly applied in the process of hyper-spectral remote sensing data.

There are two important prerequisites is the reflectance of the flat area must be close to 1 and have non absorption characteristics. In the other words, this flat area must be an absolutely diffuse reflector that can avoid the damage of the effective information caused by the very low signal to noise ratio. 
Besides, when shot the selected flat area and subject with evident difference in the shooting distance we will cannot obtain an desirable results.

\section{Dark Field Correction and Comparative Colorimetric Assay}

Even the shooting environment is totally dark, the image sensor will produce direct current in the condition of reverse bias voltage and therefore we must calibrate the camera response produced by this dark current. Dark field correction finished with lens cap or other things that can interrupt the light from the lens by subtracting the HIS response produced by dark current from the HIS response of shooting subjects.

Comparative colorimetric assay and absolute measurement method are opposites, the former measure spectral reflectance or transmission of sample on every wavelength by comparing the monochromatic radiation of a certain standard reference with known reflection characteristic and samples. This method has widely been used in various color measurement device, such as spectrophotometer colorimeter and so on. Therefore, the current measurement device must choose an absolute diffuse reflector as a standard when measure samples. But this kind of standard can't be found easily and can be replaced by the other material that has similar reflectance property with absolute diffuse reflector as white standard plate .In order to increase accuracy, the spectral reflectance of white standard plate is measured by national department of metrology via auxiliary integrating sphere or other absolute measurement method.

The calculating principle of comparative colorimetric assay as follows. Supposing the device response value and measured standard spectral reflectance of white standard plate as $C_{W}(\lambda)$ and $\mathrm{W}(\lambda)$ respectively and the device response value and the unknown spectral reflectance of sample as $C_{S}(\lambda)$ and $S(\lambda)$ respectively. Supposing the incidence radiant flux density as $\varphi_{0}(\lambda)$, the reflected radiant flux density the image sensor received of white standard plate and sample as $\varphi_{W}(\lambda)$ and $\varphi_{S}(\lambda)$ and conversion coefficient as $\mathrm{k}$.Based on the definition that reflectance equal to the ratio of reflected radiant flux density and incidence radiant flux density we can calculate the spectral reflectance of sample by the following computing process.

$$
\begin{aligned}
& \mathrm{C}_{\mathrm{S}}(\lambda)=\mathrm{k} * \varphi_{S}(\lambda)=k * \varphi_{0}(\lambda) * S(\lambda) \\
& \mathrm{C}_{\mathrm{W}}(\lambda)=\mathrm{k} * \varphi_{W}(\lambda)=k^{*} \varphi_{0}(\lambda) * W(\lambda)
\end{aligned}
$$

Each side of equation (1) divided by each side of equation (2) can obtain the equation (3) calculating the spectral reflectance of samples.

)

$$
\mathrm{S}(\lambda)=\frac{\mathrm{C}_{S}(\lambda)}{\mathrm{C}_{\mathrm{W}}(\lambda)} * W(\lambda)
$$

Supposing the device response value caused by dark current as $C_{D}(\lambda)$ we can get the calibrated spectral reflectance equation shown in the equation (4):

$$
\mathrm{S}(\lambda)=\frac{\mathrm{C}_{S}(\lambda)-\mathrm{C}_{\mathrm{D}}(\lambda)}{\mathrm{C}_{\mathrm{W}}(\lambda)-\mathrm{C}_{\mathrm{D}}(\lambda)} * W(\lambda)
$$


Combining the dark field correction and comparative colorimetric assay to reconstruct the sample's spectral reflectance from HIS image, we just need the device response value of white standard plate, samples and dark current and the absolute spectral reflectance.

\section{Experimental Verification}

\section{Experimental Condition}

The experiment in this paper use the HIS from Isuzu Optics, color checker CLASSIC from X-rite, spectrophotometer il-Pro.The Isuzu Optics HIS is comprise of spectrum camera, movable objective table, lighting source, two fixators of linear source and camera obscura and the spectrum camera is made up of lens, grating spectrograph and camera with high photo sensibility that have a $2.8 \mathrm{~nm}$ sample wavelength interval and $360-1000 \mathrm{~nm}$ wavelength range. The HIS and color checker CLASSIC as shown in the Figure 1 and Figure 2 respectively.

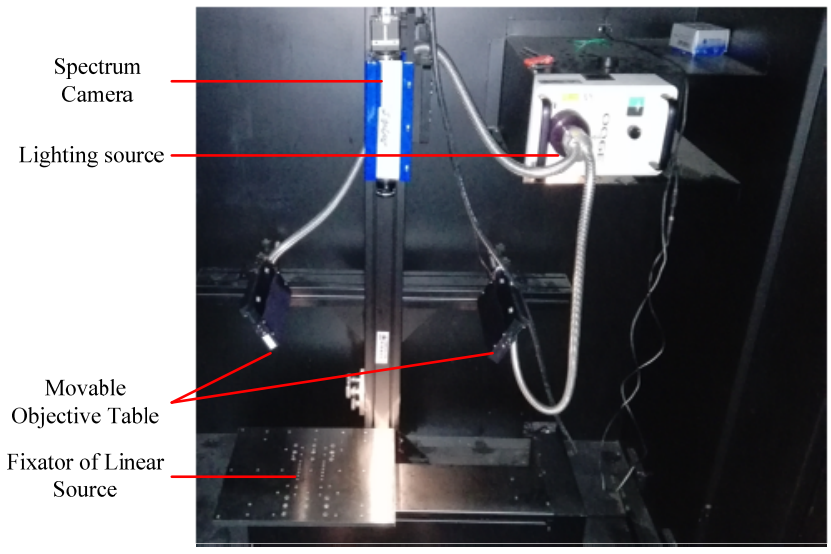

Figure.1 HIS Structure

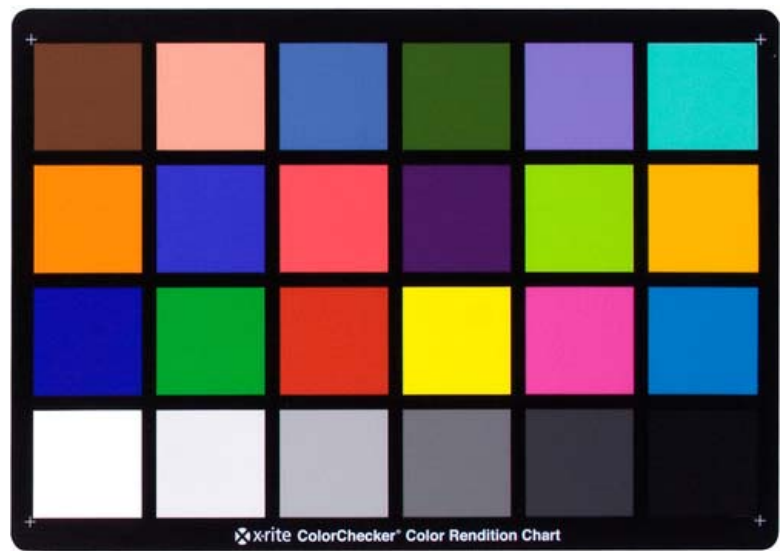

Figure.2 Color checker CLASSIC

\section{Experimental Procedure}

We captured the images of samples with the matching image capture software named Hyper Spectral Image and the specific procedure as shown in the figure 3.Lighting source adjustment can adjust the intensity and aggregation degree of lighting and be realized by adjusting the light condenser located in the two fixators of linear source to gather the light until the light getting into the movable objective table becoming light strip. Since the bit depth of this system is 12 , adjusting the Max DN to 3276 around to avoiding the signal of captured image slop over the $0 \sim 65535$. Besides, when we shot the color checker CLASSIC and white standard plate the shooting plane must be maintain at the same high level to getting the same lighting condition.

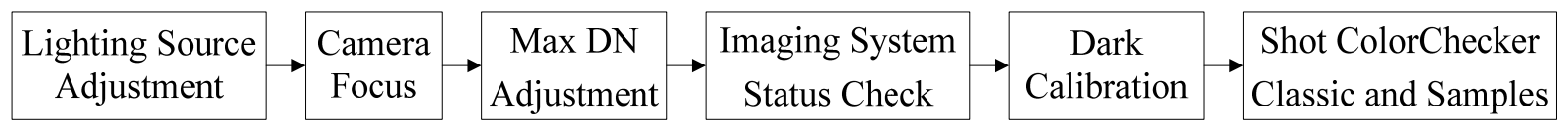

Figure.3 The Experiment Procedure

\section{HIS Status Check}

The stability of HIS: shooting the white standard plate after turning on the HIS $5,10,15,20,25,30,40,50,60$ minutes and export the image data at the same area. Calculating the range and mean value of each set of data and we found that the difference of each set data is $2 \%$ around, therefore the HIS can be regard as a stable image capture system.

The Linearity of HIS: changing the exposure time to control the incident radiation density of the image sensor and shooting the white standard plate as a set of images, then getting the HIS response 
value at the same pixel in the set of images. The result indicated that the incidence radiation flux density is linear with the HIS response value.

\section{Experiment Results Analysis}

Reconstructing the spectral reflectance of color checker CLASSIC with flat-field and comparative colorimetric assay respectively after reading the image data and calibrating the dark current and evaluating the spectrum reconstruction accuracy with two parameters named root-mean-square error(RMSE) and color aberration $(\Delta E)$. The RMSE can reflect the spectrum comparability of the

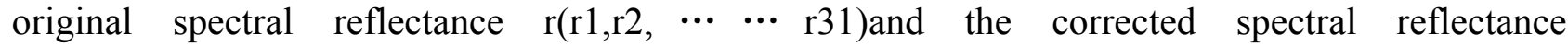
$\mathrm{p}(\mathrm{p} 1, \mathrm{p} 2, \cdots \cdots, \mathrm{p} 31)$.The $\Delta E\left(2^{\circ}, \mathrm{D} 50\right)$ can reflect the difference between original chromaticity $\mathrm{L}$ 、 $a^{*}, b^{*}$ and the corrected chromaticity L1、a1*, b1*.Besides, we use the standard deviation to evaluated the uniformity of RMSE and $\Delta E$ of color checker CLASSIC. The computational equation of RMSE and $\Delta E$ as shown following:

$$
\begin{aligned}
& \text { RMSE }=\sqrt{\frac{1}{\mathrm{n}} \sum_{i=1}^{31}\left(\mathrm{r}_{i}-p_{i}\right)^{2}} \\
& \Delta E=\sqrt{\left(L-L_{1}\right)^{2}+\left(a^{*}-a_{11}^{*}\right)^{2}+\left(b^{*}-b_{1}^{*}\right)^{2}}
\end{aligned}
$$

The RMSE and $\Delta E$ of flat-field and Comparative Colorimetry as shown in the Table1.

Table.1 The Accuracy of Spectrum Reconstruction

\begin{tabular}{|c|c|c|c|c|c|c|}
\hline \multirow{2}{*}{ Construction method } & \multicolumn{3}{|c|}{ RMSE } & \multicolumn{3}{c|}{$\Delta E$} \\
\cline { 2 - 7 } & Mean & Max & $\begin{array}{c}\text { Standard } \\
\text { Deviation }\end{array}$ & Mean & Max & $\begin{array}{c}\text { Standard } \\
\text { Deviation }\end{array}$ \\
\hline Flat-field & 0.0525 & 0.1127 & 0.0384 & 5.11 & 10.68 & 3.14 \\
\hline $\begin{array}{c}\text { Comparative } \\
\text { Colorimetric Assay }\end{array}$ & 0.0262 & 0.0647 & 0.0157 & 3.37 & 8.33 & 1.88 \\
\hline
\end{tabular}

As shown in the table above,comparing comparative colorimetric assay with Fat-Field, RMSE and $\Delta E$ decreased by $50 \%$ and $34 \%$ respectively. From contrastive analysis the standard deviation we can see that comparative colorimetric assay increased the uniformity of all the samples in color checker CLASSIC. In order to observe the compare result, we chosen the patch A3 and C4 and draw the sepctrum curve collation map as shown figure 4.
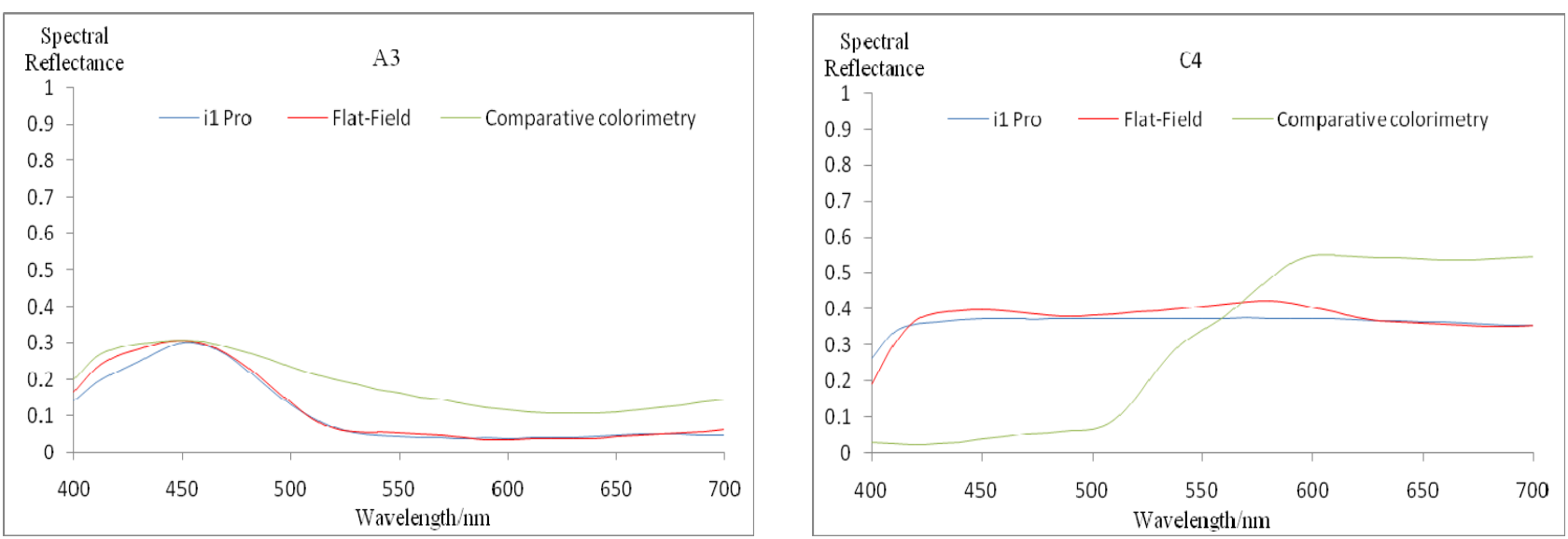

Figure.4 The Sepctrum Curve Collation Map 


\section{Conclusion}

The spectral reflectance reconstructed by flat-field is relative, but the absolute spectral reflectance is more broadly applied to the field of $\mathrm{Hi}-\mathrm{Fi}$. This paper proposed to use comparative colorimetric assay method and dark field correction in the HIS to reconstruct the absolute spectral reflectance after ensuring the system keep stable and linear. The experiment and analysis result indicated that this method is superior to the common method applied in the HIS whether spectral comparability or colorimetric and this method is more practical in the field of Hi-Fi.

\section{References}

[1]Fan DongJun, Zhang ShaoHua, Research on the Methods of the Reflectivity Inversion of the High Spectrum Image, Hydrographic Surveying and Charting. 26(2006) 28-30.

[2]Dong LianFeng, Study on the Hyperspectral Image Preprocessing, XiAn, 2007.

[3]Wang Ying,A Study of Key Technologies in Multispectral Image Color Reproduction, XiAn 2010.

[4]Yang Ping, Liao NingFang, He Li, Study on Image Acquisition and Spectral Construction Based on Speedy Multi-spectral Imaging System, Image Technology 6(2007) 14-18.

[5]Hu WeiJie, Tang ShunQing,Zhu ZhengFang,Modern Color Science and Application, BeiJing, 2007.

[6] Zhao ZiQi, Study on The Capture and Spectral Reflectance Reconstruction of Multispectral Image, RiZhao, 2014. 